\title{
Classification of metallic glasses based on structural and dynamical heterogeneities by stress relaxation
}

\author{
Zhen Lu, Wei-Hua Wang and Hai-Yang Bai*
}

\begin{abstract}
We show that the diverse dynamic responses of viscosity of the supercooled liquid near the glass transition temperature can be inherited to glassy state and expressed in the discrepancy of stress relaxation, demonstrating a structural heterogeneous basis for fragility. The metallic glasses with diverse dynamics and structural heterogeneities manifesting in different densities and distributions of flow units can be classified and characterized in terms of a parameter $n$, which can be readily determined through stress relaxation method and is comparable to the kinetic fragility $m$ of the supercooled liquid. The parameter can classify diverse metallic glasses and also benefits for understanding the correlation between the structural and dynamical heterogeneities of the metallic glasses.
\end{abstract}

Glassy state with unique and fascinating mechanical, dynamic and thermodynamic properties, which can be achieved by quenching from highly viscous liquid [1-4], is a universal property of condensed matter that lose its ability to flow. One of the most confusing issues in the glasses is that the viscosity $(\eta)$ of a glass-forming liquid can vary by several orders of magnitude in a narrow temperature range near the glass transition temperature $\left(T_{\mathrm{g}}\right)$ [5]. Based on the various responses of viscosity $\eta$ to temperature, the kinetic fragility $m$ is defined in terms of the shear viscosity $\eta$ as [6-7]:

$$
m=\left.\frac{\partial \log \eta(T)}{\partial\left(T_{\mathrm{g}} / T\right)}\right|_{T=T_{\mathrm{g}}} .
$$

The $m$, which is an index to characterize how fast the viscosity of glass-forming liquid increases with decreasing temperature while temperature $(T)$ approaching $T_{\mathrm{g}}$, directly relates to the slowing down of the dynamics of the glass-forming liquids [6]. According to the concept of fragility, the glass-forming liquids can be roughly classified into two groups: strong and fragile liquids [6]. This classification attempts to construct a relation between the macroscopic transport property close to $T_{\mathrm{g}}$ and the dynamics of glassy systems. After decades of researches, it has been found that the fragility can be traced back to dynamic and structural heterogeneities, topographic differences of the energy landscape $[7,8]$. Some features and physical properties of the glass-forming liquids or the resultant glasses can also be correlated to the kinetic fragility, such as the temperature behavior of the shear elastic modulus [9], the specific heat jump at $T_{\mathrm{g}}[10,11]$, Poisson's ratio [12], the glass-forming ability [13], and the vibrational properties of glasses far below $T_{\mathrm{g}}$ [14]. Compared with other glasses such as polymer and oxide glasses, the supercooled liquid region of the metallic alloys is narrow and lack of thermal stability $[15,16]$, the value of kinetic fragility $m$ of the metallic glass-forming liquids is hard to be accurately detected, and only few compositions have the accurate values of fragility. Therefore, it is useful and important to find an accurate parameter to classify the metallic glasses far below $T_{\mathrm{g}}$.

Recently, both computer simulations and experiments show that the metallic glasses (MGs) are of nanoscale structural heterogeneity and contain unstable and high mobility nano-scale liquid-like regions which act as flow units in the glassy state [17-21]. The nanoscale structural heterogeneity is found to relate closely to the dynamic heterogeneity (which can be reflected by the kinetic fragility $m$ ) of the metallic glass-forming liquids [17-21] and the viscoelasticity localized flow phenomenon in MGs activated by either external stress or temperature (corresponding to the deformation or initiation of the glass transition, respectively) [17-21]. The stress relaxation is found to be an effective method which can sensitively characterize and classify the microstructural heterogeneity and reveal the relaxation kinetics of the flow units of MGs below $T_{\mathrm{g}}$ $[19,22,23]$, and this method could be applied to accurately and readily classify the MGs based on their discrepancy in microstructural heterogeneity which closely related to the dynamic heterogeneity of MGs.

In this letter, we apply an instantaneous activation-relaxation technique to investigate the stress relaxation behavior of a variety of metallic glasses. We show that in MGs, the different responses of viscosity to temperature in the liquid state could be reserved in the glass state and is manifested in different structural heterogeneities which can be reflected by using stress relaxation behaviors. We define a 
parameter $n$ which is equivalent to kinetic fragility $m$ of the supercooled liquid but much easier to be experimentally determined in the metallic glassy state using stress relaxation method. We show that the parameter $n$ can readily classify the glasses with various structural heterogeneities far below $T_{\mathrm{g}}$ and correlate with some properties of the MGs.

We used a series of MGs and polymer which have different values of kinetic fragility (their index of fragility are listed in Table 1). The metallic ingots with nominal compositions listed in Table 1 were prepared by arc melting from pure metallic elements and the glassy ribbons were prepared by melt-spinning method using a $\mathrm{Cu}$ wheel at a tangential velocity of $\sim 25 \mathrm{~m} \mathrm{~s}^{-1}$ in vacuum [16]. Their glassy nature of the as-spun ribbon alloys was ascertained by X-ray diffraction and differential scanning calorimeter. The stress relaxation measurements were performed on a TA DMA Q800 dynamical mechanical analyzer (DMA), and the glassy ribbon samples were mounted in tension clamp and the polymer plate was tested through 3-point bending. A constant strain of $0.6 \%$ [in the apparent elastic regime $(\sim 2 \%)$ of metallic glass $(\mathrm{MG})]$ at $0.78 T_{\mathrm{g}}$ was rapidly applied on the samples and the hold time was $120 \mathrm{~min}$ for each test to record the stress decay. Before the experiments, a 5-min delay was applied to allow the samples to equilibrate at the test temperatures. To avoid the effect of the different cooling rates, all the samples were previously heated to the supercooled liquid regions and cooled down to the room temperature in the argon atmosphere prior to the measurements. Besides, to ensure the accuracy of strain value, the length of sample was measured before and after each stress relaxation test.

Fig. 1a shows the stress relaxation response of a typi-
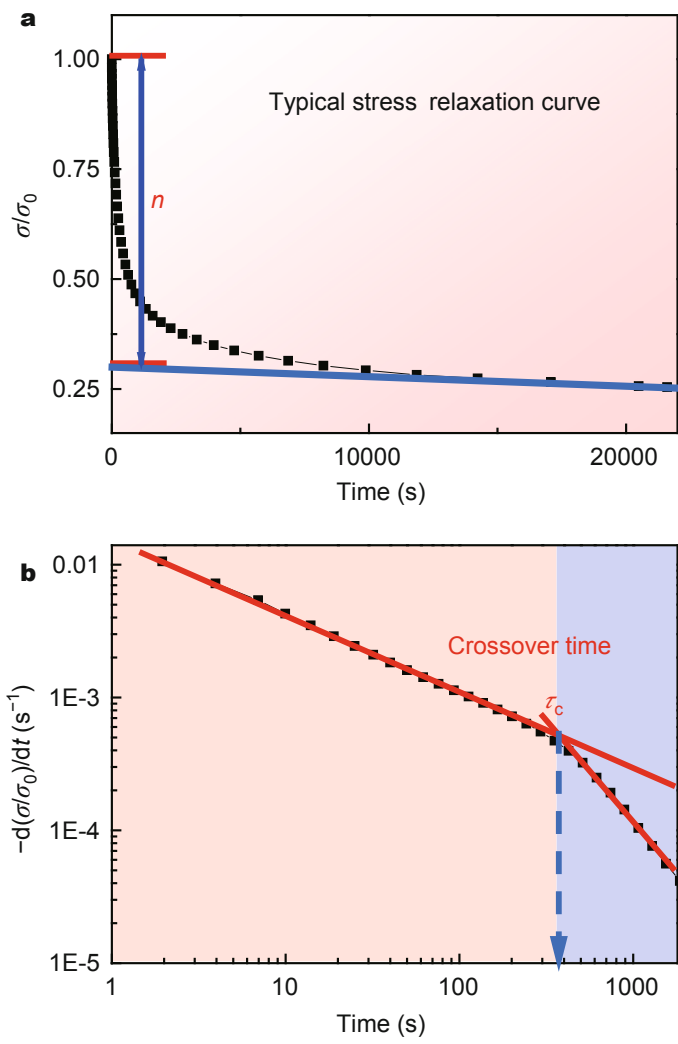

Figure 1 (a) The typical stress relaxation curve of $\mathrm{La}_{55} \mathrm{Ni}_{20} \mathrm{Al}_{25} \mathrm{MG}$. The stress is divided by initial stress. A tangent line to the stress relaxation curve at 21,600 s is drawn and extended to intersect with the $Y$ axis and the difference between 1.0 and the ordinate value of the intersection is defined as $n$. (b) The log-log plot of scaled stress reduction rate against time of $\mathrm{Gd}_{55} \mathrm{Al}_{25} \mathrm{Ni}_{10} \mathrm{Co}_{10} \mathrm{MG}$. The curve shows two parts of power law relationship, corresponding to the fast and slow relaxations and the time of crossover point is defined as $\tau_{\mathrm{c}}$.

Table 1 Comparison of the fragility, $v, n, T_{\mathrm{g}}$ and the test temperature for various MG

\begin{tabular}{|c|c|c|c|c|c|}
\hline Composition & $m$ (Ref.) & $v$ (Ref.) & $n$ & $T_{\mathrm{g}}(\mathrm{K})$ & Test temperature $(\mathrm{K})$ \\
\hline $\mathrm{Ce}_{70} \mathrm{Al}_{10} \mathrm{Cu}_{20}$ & $28[34]$ & $0.329[42]$ & 0.0851 & 382 & 298 \\
\hline $\mathrm{Ce}_{68} \mathrm{Al}_{10} \mathrm{Cu}_{20} \mathrm{Co}_{2}$ & $35[35]$ & $0.328[42]$ & 0.1076 & 350 & 273 \\
\hline $\mathrm{Ce}_{70} \mathrm{Al}_{10} \mathrm{Ni}_{10} \mathrm{Cu}_{10}$ & $37.2[13]$ & $0.313[42]$ & 0.1993 & 350 & 273 \\
\hline $\mathrm{Cu}_{46} \mathrm{Zr}_{46} \mathrm{Al}_{8}$ & $41[36]$ & $0.366[42]$ & 0.3140 & 700 & 546 \\
\hline $\mathrm{Zr}_{65} \mathrm{Cu}_{15} \mathrm{Ni}_{10} \mathrm{Al}_{10}$ & $36[37]$ & $0.355[42]$ & 0.3572 & 628 & 490 \\
\hline $\mathrm{Zr}_{65} \mathrm{Al}_{7.5} \mathrm{Cu}_{17.5} \mathrm{Ni}_{10}$ & 35 [37] & $0.355[42]$ & 0.3990 & 628 & 490 \\
\hline $\mathrm{Cu}_{45} \mathrm{Zr}_{45} \mathrm{Al}_{7} \mathrm{Gd}_{3}$ & $30[38]$ & $0.358[42]$ & 0.4613 & 649 & 506 \\
\hline $\mathrm{Zr}_{41.2} \mathrm{Ti}_{13.8} \mathrm{Cu}_{12.5} \mathrm{Ni}_{10} \mathrm{Be}_{22.5}$ & $50[37]$ & $0.353[42]$ & 0.4894 & 620 & 484 \\
\hline $\mathrm{Cu}_{50} \mathrm{Zr}_{50}$ & $62[38]$ & $0.360[42]$ & 0.5500 & 657 & 512 \\
\hline $\mathrm{Gd}_{55} \mathrm{Al}_{25} \mathrm{Cu}_{10} \mathrm{Co}_{5} \mathrm{Ni}_{5}$ & 37 [39] & - & 0.6280 & 563 & 439 \\
\hline $\mathrm{La}_{55} \mathrm{Ni}_{20} \mathrm{Al}_{25}$ & $42[40]$ & $0.348[42]$ & 0.6415 & 473 & 369 \\
\hline $\mathrm{Pd}_{40} \mathrm{Cu}_{30} \mathrm{Ni}_{10} \mathrm{P}_{20}$ & $63[37]$ & $0.399[42]$ & 0.6422 & 566 & 441 \\
\hline $\mathrm{Gd}_{55} \mathrm{Al}_{25} \mathrm{Cu}_{10} \mathrm{Co}_{10}$ & $45[39]$ & - & 0.7270 & 578 & 451 \\
\hline $\mathrm{Gd}_{55} \mathrm{Al}_{25} \mathrm{Ni}_{10} \mathrm{Co}_{10}$ & $58[39]$ & - & 0.7446 & 593 & 463 \\
\hline $\mathrm{Gd}_{55} \mathrm{Al}_{25} \mathrm{Co}_{20}$ & $74[39]$ & - & 0.7650 & 598 & 466 \\
\hline PMMA & $145[41]$ & $0.425[43]$ & 0.7830 & 428 & 334 \\
\hline
\end{tabular}


cal $\mathrm{La}_{55} \mathrm{Ni}_{20} \mathrm{Al}_{25} \mathrm{MG}$ to a constant strain of $0.6 \%$ at $369 \mathrm{~K}$ $\left(=0.78 T_{\mathrm{g}}\right)$. The stress relaxation plots were normalized by the initial stress $\sigma_{0}$. The time dependent stress $\sigma(t)$ shows a rapidly decrease from $\sigma_{0}$ in the relaxation time of $600 \mathrm{~s}$, and then obviously slows down and decays sluggishly with extended increasing time. Through the differential of scaled stress against time in a log-log fashion, the stress relaxation curve can be divided into two distinct power-law processes as shown in Fig. 1b. An obvious crossover time $\tau_{c}$ determined based on the intersection of the two power-law fittings represents the transformation of the two processes. The first fast power-law process corresponds to the stochastic activation of the localized nano-scale flow units and consumes the applied energy quickly; and the second slow power-law process corresponds to self-organized cooperative motion of the activated flow units [22]. In the fast process, a portion of the flow units with a shorter relaxation time and low activation energy are quickly activated, and then the residual flow units with higher activation energy are gradually activated with the time increasing [19]. In the slow process, the variation of scaled stress decay with time keeps the similar sluggish decay tendency even in relaxation for 21,600 s. The subsequent sluggish stress relaxation over time can be attributed to two reasons: (i) the flow units which have higher activation energy or a longer relaxation time need enough time to be activated [19]; (ii) along with the number of activated flow units increasing, the adjacent activated flow units will merge into a larger one [24].

The size, activation energy, intrinsic relaxation time and their distributions of flow units reflecting the structural heterogeneity of a glass are quite different in various MGs [22]. The different structural heterogeneities cause the difference in behaviors of stress relaxation and can be manifested in different stress attenuation degrees. Through the difference of the stress attenuation degree during the stress relaxation measurement, we can easily define a straightforward parameter ' $n$ ' to classify the MGs as shown in Fig. 1a: a tangent line along the sluggish linear stress relaxation curve at $0.78 T_{\mathrm{g}}$ is drawn and extended to intersect with the stress axis. The difference between 1.0 and the ordinate value of the intersection, which is attenuation degree of stress relaxation, is defined as fragility $(n)$ of a glassy state as indicated in Fig. 1a. The $n$ represents how fast of the process of the stochastic activation of the flow units, and can reflect the density of the flow units or structural heterogeneity in a MG, which is intrinsic nature of a glass. In the following, we will show that the parameter $n$, which is easily measured by stress relaxation method comparing with the kinetic fragility of the metallic supercooled liquids, can also accurately reflect the dynamics property and structural heterogeneity of MGs below $T_{\mathrm{g}}$.
We select a series of glasses, which have different compositions, glass-forming abilities, physical and mechanical properties, and fragility, to measure their $n$ values. Fig. 2a shows the stress relaxation curves of sixteen various glasses at $0.78 T_{\mathrm{g}}$ and constant strain of $0.6 \%$ for $7200 \mathrm{~s}$. We see that all of the stress relaxation curves present dramatic decay
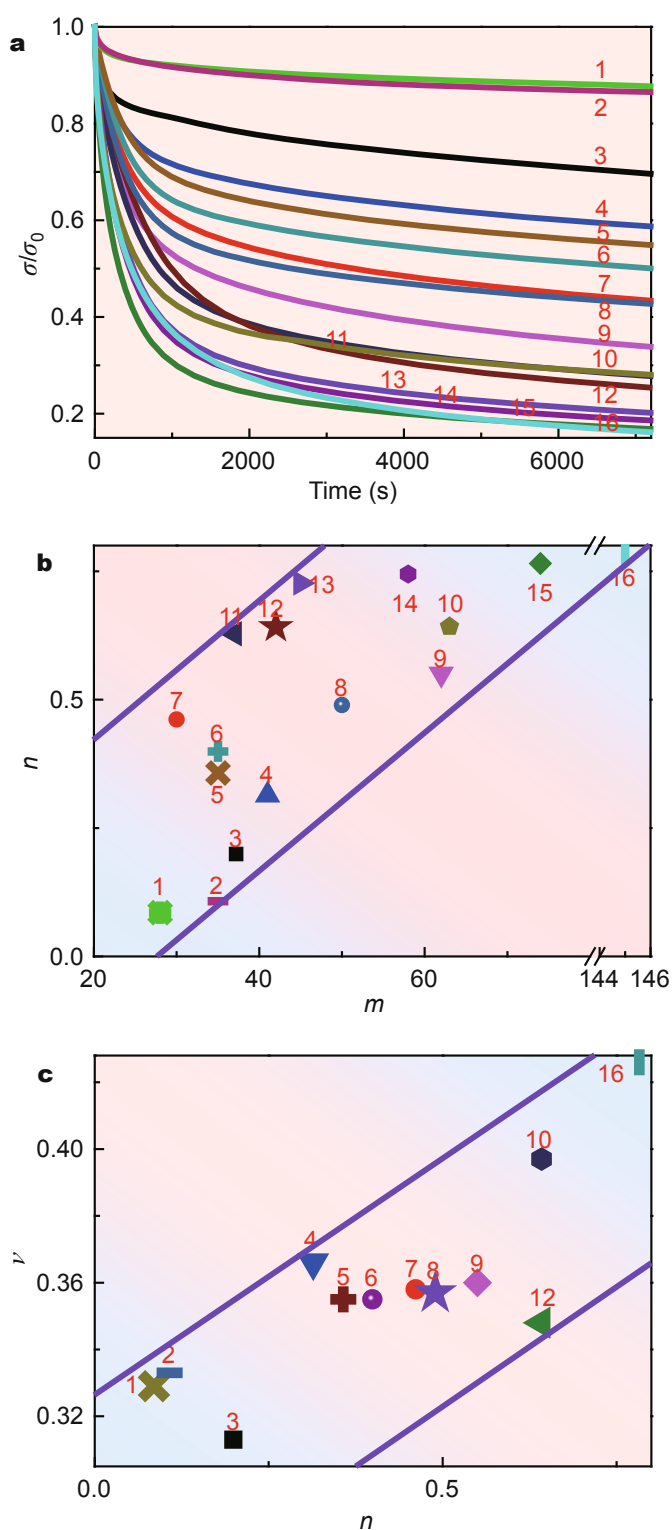

Figure 2 (a) The stress relaxation curves for 16 glasses. From 1-16, the composition of glasses is $\mathrm{Ce}_{70} \mathrm{Al}_{10} \mathrm{Cu}_{20}, \mathrm{Ce}_{68} \mathrm{Al}_{10} \mathrm{Cu}_{20} \mathrm{Co}_{2}, \mathrm{Ce}_{70} \mathrm{Al}_{10} \mathrm{Ni}_{10} \mathrm{Cu}_{10}$, $\mathrm{Cu}_{46} \mathrm{Zr}_{46} \mathrm{Al}_{8}, \mathrm{Zr}_{65} \mathrm{Cu}_{15} \mathrm{Ni}_{10} \mathrm{Al}_{10}, \mathrm{Zr}_{65} \mathrm{Al}_{7.5} \mathrm{Cu}_{17.5} \mathrm{Ni}_{10}, \mathrm{Cu}_{45} \mathrm{Zr}_{45} \mathrm{Al}_{7} \mathrm{Gd}_{3}, \mathrm{Zr}_{41.2}$ $\mathrm{Ti}_{13.8} \mathrm{Cu}_{12.5} \mathrm{Ni}_{10} \mathrm{Be}_{22.5}, \mathrm{Cu}_{50} \mathrm{Zr}_{50}, \mathrm{Pd}_{40} \mathrm{Ni}_{10} \mathrm{Cu}_{30} \mathrm{P}_{20}, \mathrm{Gd}_{55} \mathrm{Al}_{24} \mathrm{Cu}_{10} \mathrm{Co}_{5} \mathrm{Ni}_{5}, \mathrm{La}_{55}$ $\mathrm{Ni}_{20} \mathrm{Al}_{25}, \mathrm{Gd}_{55} \mathrm{Al}_{25} \mathrm{Cu}_{10} \mathrm{Co}_{10}, \mathrm{Gd}_{55} \mathrm{Al}_{25} \mathrm{Ni}_{10} \mathrm{Co}_{10}, \mathrm{Gd}_{55} \mathrm{Al}_{25} \mathrm{Co}_{20}$ and PMMA, respectively. (b) The index of fragility $m$ vs. $n$ (the solid line is a guide for eye). (c) The plot of the Poisson's ratio $v$ vs. $n$ (the solid line is a guide for eye). The sequencing numbers of the glasses are consistent with those in Fig. 2a. 
before $1000 \mathrm{~s}$, but different glasses show markedly different attenuation degrees. After about $1000 \mathrm{~s}$, the decay for all the glasses tends toward a slow process in a long time scale. The various attenuation degrees in their fast decay process reflect the different heterogeneity microstructure or different size, intrinsic relaxation time and distribution of flow units in MGs inheriting from the dynamic heterogeneity in their liquid state which can be characterized by the kinetic fragility. We determined the $n$ values of these MGs, and compared the $n$ values with their kinetic fragility $m$ obtained from the literatures. Currently, there are not so many MG formers whose kinetic fragility are known due to the experimental difficulties for directly measuring their viscosity change of their supercooled liquid state approaching $T_{\mathrm{g}}$. And the values of $m$ for these MGs are mostly indirectly measured by a purely thermodynamic way using calorimetric methods, which is hard to reflect their relaxation behaviors in liquid state and inevitably contains large ambiguity [25,26].

Fig. $2 \mathrm{~b}$ shows the comparison of the obtained $n$ values with the values of fragility $m$ of the various MGs. The relatively broad distribution of the plot of fragility $m v s . n$ is due to that the original data of $m$ of these MGs are obtained under a variety of different groups with various testing conditions by different methods in their glassy state. Even though the large ambiguity of the values of $m$ causes the broad distribution of $n v s$. $m$, one can see that the $n$ and $m$ show a rough correlation. The fragile MGs with the larger $m$ show more rapidly decrease of stress relaxation, corresponding to the larger values of $n$. The stress relaxation of strong MGs reaches the constant value more quickly and shows smaller $n$. The comparison indicates the similarity and correlation between the $n$ and $m$. The thermodynamic, dynamic and mechanical properties of polymer differ from that of MGs and show the broad distribution of fragility. To prove the stress relaxation is a universal method to classify glasses, we also tested the relaxation behavior of a typical glassy polymer of poly-methylmethacrylate (PMMA) as shown in Fig. 2b. The fragility index of PMMA with the largest value of $m(m=145)$ shows the maximum attenuation degree. It is worth noting that the La-based MGs with obvious $\beta$-relaxation around the $0.78 T_{\mathrm{g}}$ show obvious relaxation behavior, even though these MGs do not have large fragility value, and this is due to that the $\beta$-relaxation has been activated around the stress relaxation temperature of $0.78 T_{\mathrm{g}}$ and leads to significant relaxation [19,27].

The relative strength of shear and bulk moduli, or Poisson's ratio $(v)$, which is the relative transverse expansion divided by the relative compression in the direction of an applied force and the fundamental property of glass, correlates with mechanical behavior of a glass such as toughness, plasticity or brittleness [28]. Fig. 2c presents the re- lationship between Poisson's ratio $v$ and $n$. The MGs with $v<0.31-0.32$ are brittle and exhibit inconspicuous stress attenuation degree [28]. With the Poisson's ratio increasing, the MGs transfer from brittle to ductile, and the stress relaxation degree of MGs increases gradually and values of $n$ become larger. This indicates a correlation between Poisson's ratio and the stress relaxation attenuation degree or $n$. The results might suggest that the microstructural origin of the Poisson's ratio and plasticity criterion [28] in various MGs is due to the degree of the heterogeneity or the density of the flow units which can be characterized by the parameter $n$ : the MGs with larger values of $n$ show a larger Poisson's ratio and better plasticity. The Poisson's ratio is found to correlate with kinetic fragility [12], which implies that the fragility coming from the viscous characteristics of the liquid phase is frozen into the glass state and can be manifested by the changing of the Poisson's ratio [12]. However, concerns have been raised on the correlations, and the reason is that there are not so many MG formers, whose kinetic fragility and elastic constants are all known, on the other hand, the calorimetric fragility is usually calculated at a higher and not well defined glass transition temperature $T_{\mathrm{g}}$. This introduces an additional source of uncertainty. Since the stress relaxation is a sensitive method to detect the structural heterogeneity of glasses that inherites from dynamic heterogeneous of liquid state, the parameter $n$ obtained by this method is expected to check the correlation and to yield information about the relation of the structural heterogeneity of the glasses and the dynamic heterogeneous of their liquid states.

Angell plot is a useful classification of liquids along a 'strong' to 'fragile' scale. The viscosity or relaxation time of strong liquids behaves in nearly Arrhenius behavior, whereas fragile liquids show conspicuous deviation from Arrhenius fashion [4]. In analogy to Angell plot, we can also obtain the similar stress relaxation plot with time for various metallic glasses as shown in Fig. 3. We use $\left(\sigma-\sigma_{\mathrm{c}}\right) /$ $\left(\sigma_{0}-\sigma_{\mathrm{c}}\right)$ to make the initial and destination coordinates of the stress relaxation curves converged into the same point (the $\sigma_{\mathrm{c}}$ is the stress value at crossover time), and the time is scaled with the crossover time $\tau_{\mathrm{c}}$ in the relaxation behavior. The $\tau_{\mathrm{c}}$ has a broad distribution for different glasses with different compositions, and the glasses can be divided by crossover time $\tau_{\mathrm{c}}$ in the relaxation behavior by reason that the time window for stochastic activation of flow units is closely related to the fragility [23]. One can see from Fig. 3 that the stress relaxation curve of fragile glasses shows obvious larger curvature compared with that of the strong one, which is similar to the Angell plot. This further confirms that the discrepancy of glasses below $T_{\mathrm{g}}$ could be distinguished through stress relaxation method by the definition of the $n$ parameter and the $n$-plot. 


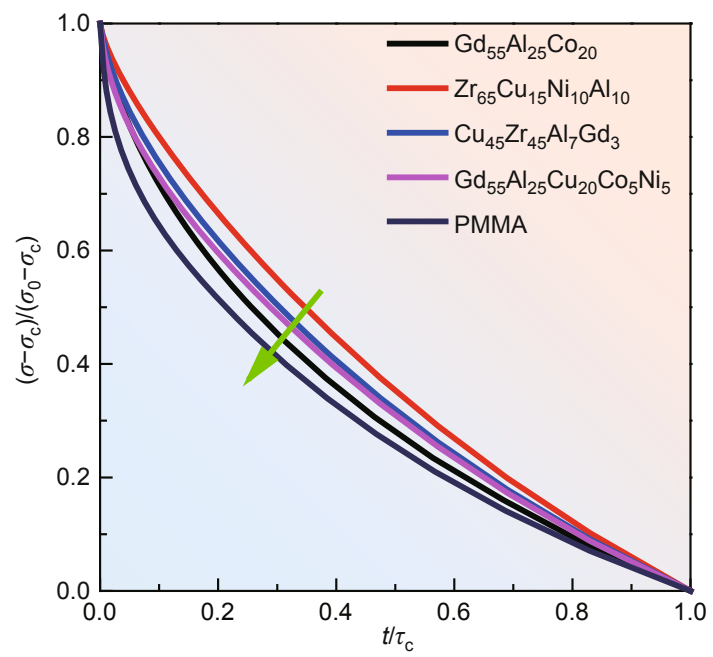

Figure 3 The plot of stress relaxation against the normalized time. The fragile glasses show obvious curvature.

We obtained the distribution of activation energy of flow units in MGs by using the activation energy spectrum model [29]. The activation energy of the relaxation processes which are available to contribute to the observed change in property on relaxation is distributed over a continuous spectrum. The measured property change, $\delta P$ can be written as:

$$
\delta P=p(E) \mathrm{d} E,
$$

where $p$ is the property change related to the relaxation processes having activation energy in the range $E$ to $E+\mathrm{d} E$. The total change in the stress relaxation $\Delta \sigma$ is given by:

$$
\Delta \sigma=\int_{0}^{\infty} p(E) \theta(E, T, t) \mathrm{d} E,
$$

where $\Delta \sigma(t)=\sigma_{0}-\sigma(t), \sigma(t)$ is the stress obtained with relaxation time $t, \sigma_{0}$ is the initial stress and $\theta(E, T, t)$ is defined as the characteristic annealing function. The function $\theta(E$, $T, t)=1-\exp (-t / \tau)=1-\exp \left[-v_{0} t \exp (E / k T)\right]$ is a measure of the proportion of available processes at energy $E$, which has contributed to the property relaxation after time $t$ at the test temperature, T. $v_{0}$ is the Debye frequency $10^{13} \mathrm{~s}^{-1}$. The $E_{\mathrm{FU}}$ has a broad distribution and only those flow units with $E_{\mathrm{FU}}<E_{0}$ have contribution to the relaxations. $E_{0}$ is critical energy for activation of a flow unit. In the frame of step-like approximation, Equation (3) can be rewritten as:

$$
\Delta \sigma=\int_{0}^{E_{0}} p(E) \mathrm{d} E
$$

where $E=k T \ln \left(v_{0} t\right)$. Then the distribution of $p(E)$ can be calculated through Equation (4) as:

$$
p(E)=-\frac{1}{k T} \frac{\mathrm{d} \sigma(t)}{\mathrm{d} \ln t} .
$$

Fig. $4 \mathrm{a}$ is the activation energy spectra $p(E)$ of the flow units induced by all the activation processes in the energy range of $E$ to $E+\mathrm{d} E$ of various MGs. The activation energy is divided by the activation energy $E_{c}$, where its relaxation time is equal to $\tau_{c}$. From the $p(E)$ of the flow units, the relative concentration of the activated flow units can be obtained via integrating the activation energy spectra. Fig. $4 \mathrm{~b}$ presents the relationship between the relative concentration of the activated flow units and $n$ for different MGs. It can be seen that the total number of activation flow units shows a close relationship with the value of $n$. The fragile MGs such as $\mathrm{Gd}_{55} \mathrm{Al}_{25} \mathrm{Co}_{20}$ shows larger value of $n$ and contains the largest number of activation flow units. These results confirm that the parameter $n$ can reflect the degree of structural heterogeneity and concentration of potential flow units in a MG, and can then classify the MGs.

We note that the amplitude and relaxation rate of stress relaxation have correlation with the test temperature. The higher relaxation temperature could activate more flow units and lead to faster and more complete relaxation [22]. Fig. 5 presents the value of $n$ changes over temperature for three MGs. It is found that the $n$ increases monotonously with temperature increasing and approximately follows a
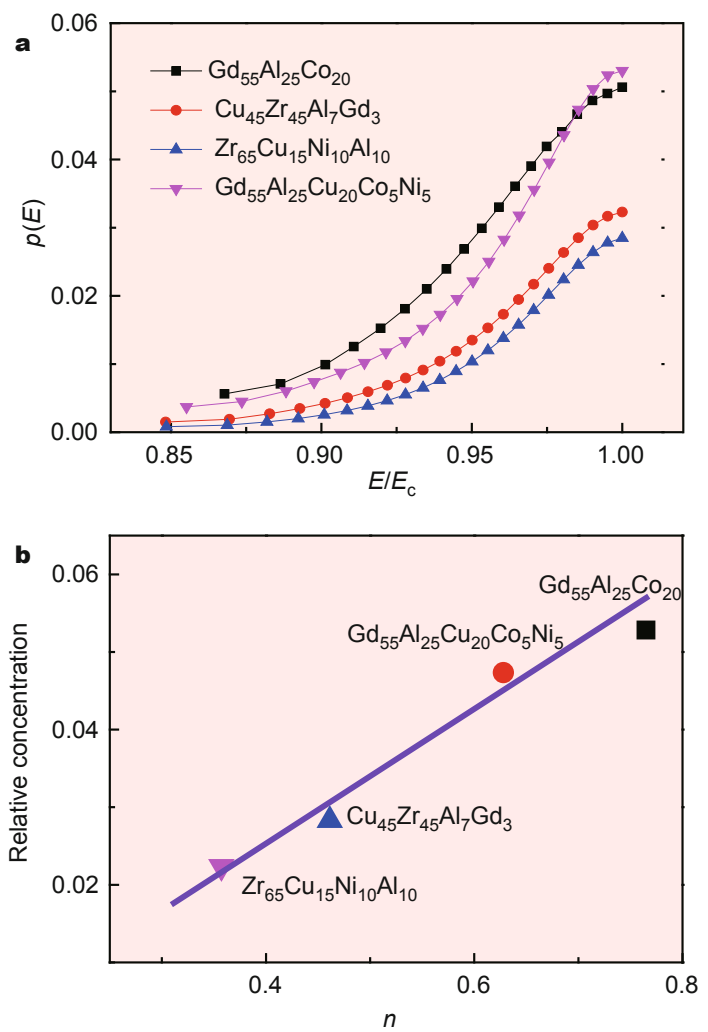

Figure 4 (a) Activation energy spectra for four MGs. (b) The total number density of activation flow units (the concentration is a relative value) and the solid line is a guide for eye. 


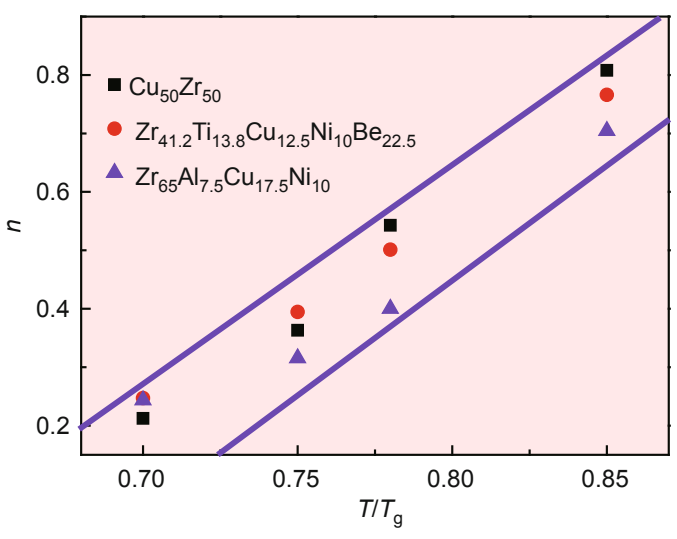

Figure 5 The $n$ values of three MGs shows the same tendency with the increase of temperature.

linear relationship for all the MGs. This indicates that even the value of $n$ depends on the test temperature, the relative variation of $n$ remains about the same for the same test temperature of $T / T_{\mathrm{g}}$. Here, we select the test temperature of $0.78 T_{\mathrm{g}}$ because we can gain obvious stress relaxation in reasonable test time scale and make clear distinctions among several MGs simultaneously.

The relation between the fragility and behavior of stress relaxation could be explained in the view of energy landscape theory: the energy landscape of strong glass consists of few megabasins, whereas fragile ones display a proliferation of well-separated megabasins in its energy landscape [5]. Because the energy landscapes of fragile MGs is more complex than that of the strong MGs, the flow units in fragile glass have higher density, and can be easily activated, and the flow units can easily sample new minimum state in energy landscape and generate more rapidly stress relaxation $[30,31]$.

The fragility of MGs reflects the viscosity of a fluid varying with the inverse temperature, and the stress relaxation is the relaxation behavior of the frozen microstructure from liquid state in the glass. The found similarity between the liquid nature of fragility $m$ and the degree of the stress relaxation $n$ in glassy state which has a characteristic timescales more than 12 orders of magnitude indicates the properties and dynamic heredity between the glass-forming liquid and glass state. That is, the liquid property can leave an unexpected 'fingerprint' frozen into the glass [32], and the dynamic inhomogeneity in glasses can be inherited from liquid and frozen into structural heterogeneity in glass state [33]. The fragile liquid with highly dynamic inhomogeneity can be quenched into a glass with high density of structural inhomogeneity or high density of flow units. Through applying stress or raising temperature, the flow units, which reflect the density of the structural inhomogeneity inherited from the dynamic inhomogeneity of the liquid, will be activated and consume energy in varying degrees for different MGs and reflect the connection with fragility. Comparing with the concept of kinetic fragility of the liquid, the parameter $n$ obtained from the stress relaxation is a parameter which can more effectively classify the metallic glassy state.

In conclusion, we propose the parameter $n$ which can be easily determined by stress relaxation to classify MGs in their glass state. We demonstrate that the parameter $n$ or $n$-plot can reflect the intrinsic feature and the density of potential flow units or the structural heterogeneity of various metallic glasses. The large ambiguity of the value of kinetic fragility $m$ for the liquid determined in glassy state has caused the arguments on the correlation between $m$ and other features and properties in glasses. Further work with the parameter $n$ can be extended for studying the correlation of $n$ with the glassy features and properties, which are crucial for understanding the nature and designing novel glasses.

Received 12 January 2015; accepted 26 January 2015 published online 5 February 2015

1 Schroers J. Bulk metallic glasses. Phys Today, 2013, 66: 32-37

2 Klement W, Willens RH, Duwez POL. Non-crystalline structure in solidified gold-silicon alloys. Nature, 1960, 187: 869-870

3 Ito K, Moynihan CT, Angell CA. Thermodynamic determination of fragility in liquids and a fragile-to-strong liquid transition in water. Nature, 1999, 398: 492-495

4 Mauro NA, Blodgett M, Johnson ML, Vogt AJ, Kelton KF. A structural signature of liquid fragility. Nat Commun, 2014, 5: 4616

5 Debenedetti PG, Stillinger FH. Supercooled liquids and the glass transition. Nature, 2001, 410: 259-267

6 Angell CA. Formation of glasses from liquids and biopolymers. Science, 1995, 267: 1924-1935

7 Angell CA. Spectroscopy simulation and scattering, and the medium range order problem in glass. J Non-Cryst Solids, 1985, 73: 1-17

8 Stillinger FH. A topographic view of supercooled liquids and glass formation. Science, 1995, 267: 1935-1939

9 Dyre JC, Olsen NB. Landscape equivalent of the shoving model. Phys Rev E, 2004, 69: 042501

10 Ngai KL, Yamamuro O. Thermodynamic fragility and kinetic fragility in supercooling liquids: a missing link in molecular liquids. J Chem Phys, 1999, 111: 10403

11 Huang D, McKenna GB. New insights into the fragility dilemma in liquids. J Chem Phys, 2001, 114: 5621-5630

12 Novikov VN, Sokolov AP. Poisson's ratio and the fragility of glass-forming liquids. Nature, 2004, 431: 961-963

13 Park ES, Na JH, Kim DH. Correlation between fragility and glassforming ability/plasticity in metallic glass-forming alloys. Appl Phys Lett, 2007, 91: 031907

14 Scopigno T, Ruocco G, Sette F, Monaco G. Is the fragility of a liquid embedded in the properties of its glass? Science, 2003, 302: 849-852

15 Greer AL. Metallic glasses. Science, 1995, 267: 1947-1953

16 Wang WH. Bulk metallic glasses with functional physical properties. Adv Mater, 2009, 21: 4524-4544

17 Ye JC, Lu J, Liu CT, Wang Q, Yang Y. Atomistic free-volume zones and inelastic deformation of metallic glasses. Nat Mater, 2010, 9: 619-623

18 Dmowski W, Iwashita T, Chuang CP, Almer J, Egami T. Elastic het- 
erogeneity in metallic glasses. Phys Rev Lett, 2010, 105: 205502

19 Lu Z, Jiao W, Wang WH, Bai HY. Flow unit perspective on room temperature homogeneous plastic deformation in metallic flasses. Phys Rev Lett, 2014, 113: 045501

20 Peng HL, Li MZ, Wang WH. Structural signature of plastic deformation in metallic glasses. Phys Rev Lett, 2011, 106: 135503

21 Liu YH, Wang D, Nakajima K, et al. Characterization of nanoscale mechanical heterogeneity in a metallic glass by dynamic force microscopy. Phys Rev Lett, 2011, 106: 125504

22 Jiao W, Wen P, Peng HL, et al. Evolution of structural and dynamic heterogeneities and activation energy distribution of deformation units in metallic glass. Appl Phys Lett, 2013, 102: 101903

23 Jiao W, Sun BA, Wen P, et al. Crossover from stochastic activation to cooperative motions of shear transformation zones in metallic glasses. Appl Phys Lett, 2013, 103: 081904

24 Krisponeit JO, Pitikaris S, Avila KE, et al. Crossover from random three-dimensional avalanches to correlated nano shear bands in metallic glasses. Nat Commun, 2014, 5: 3616

25 Samwer K, Löhneysen $\mathrm{H}$. Amorphous superconducting $\mathrm{Zr}_{x} \mathrm{Cu}_{1-x}$ : electronic properties, stability, and low-energy excitations. Phys Rev B, 1982, 26: 107-123

26 Wang WH. Correlations between elastic moduli and properties in bulk metallic glasses. J Appl Phys, 2006, 99: 093506

$27 \mathrm{Yu} \mathrm{HB}$, Shen X, Wang Z, et al. Tensile plasticity in metallic glasses with pronounced $\beta$ relaxations. Phys Rev Lett, 2012, 108: 015504

28 Lewandowski JJ, Wang WH, Greer AL. Intrinsic plasticity or brittleness of metallic glasses. Phil Mag Lett, 2005, 85: 77-87

29 Gibbs MRJ, Evetts JE, Leake JA. Activation energy spectra and relaxation in amorphous materials. J Mater Sci, 1983, 18: 278-288

30 Bonn D, Tanase S, Abou B, Tanaka H, Meunier J. Laponite: aging and shear rejuvenation of a colloidal glass. Phys Rev Lett, 2002, 89: 015701

31 Sastry S, Debenedetti PG, Stillinger FH. Signatures of distinct dynamical regimes in the energy landscape of a glass-forming liquid. Nature, 1998, 393: 554-557

32 Dyre JC. Glasses: heirs of liquid treasures. Nat Mater, 2004, 3: $749-750$

33 Cooper W, Harrowell AP, Fynewever H. How reproducible are dynamic heterogeneities in a supercooled liquid? Phys Rev Lett, 2004, 93: 135701
34 Wang WH. Correlation between relaxations and plastic deformation, and elastic model of flow in metallic glasses and glass-forming liquids. J Appl Phys, 2011, 110: 053521

35 Zhang B, Wang RJ, Zhao DQ, Pan MX, Wang WH. Properties of Ce-based bulk metallic glass-forming alloys. Phys Rev B, 2004, 70: 224208

36 Zhao ZF, Wen P, Shek CH, Wang WH. Measurements of slow $\beta$-relaxations in metallic glasses and supercooled liquids. Phys Rev B, 2007, 75: 174201

37 Fan GJ, Choo H, Liaw PK. Fragility of metallic glass-forming liquids: a simple thermodynamic connection. J Non-Cryst Solids, 2005, 351: 3879-3883

38 Li Y, Bai HY, Wang WH, Samwer K. Low-temperature specific-heat anomalies associated with the boson peak in CuZr-based bulk metallic glasses. Phys Rev B, 2006, 74: 052201

39 Jing G, Bian XF, Zhao Y, et al. Correlation between the fragility of supercooled liquids and thermal expansion in the glassy state for Gd-based glass-forming alloys. J Phys Cond Matter, 2007, 19: 116103

40 Senkov ON. Correlation between fragility and glass-forming ability of metallic alloys. Phys Rev B, 2007, 76: 104202

41 Roland CM, Santangelo PG, Ngai KL. The application of the energy landscape model to polymers. J Chem Phys, 1999, 111: 5593-5598

42 Wang WH. The elastic properties, elastic models and elastic perspectives of metallic glasses. Prog Mater Sci, 2012, 57: 487-656

43 Lu H, Zhang X, Knauss WG. Uniaxial, shear, and poisson relaxation and their conversion to bulk relaxation: studies on poly(methyl methacrylate). Polym Eng Sci, 1997, 37: 1053-1064

Acknowledgements Experimental assistance with Yanzhuo Li, Yitao Sun, Ping Wen, Deqian Zhao, Dawei Ding, Zhiguang Zhu, Meng Gao, and Baoan Sun are appreciated. This work was supported by the National Natural Science Foundation of China (51271195) and the National Basic Research Program of China (2015CB856800).

Author contributions $\mathrm{Lu} \mathrm{Z}$ conducted the experiments and analyzed the data. Bai HY and Wang WH supervised the experiments and analysis. Lu Z, Wang WH and Bai HY wrote the paper together.

Conflict of interest The authors declare that they have no conflict of interest. 


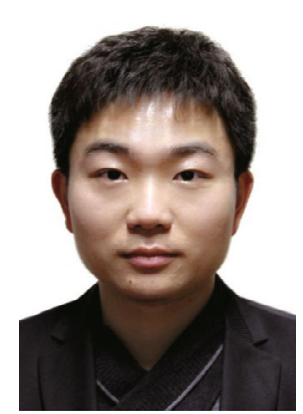

Zhen Lu is a PhD candidate in condensed matter physics at the Institute of Physics, Chinese Academy of Sciences at Beijing, China, supervised by Professor Hai-Yang Bai. His research focuses on the relaxation behavior in metallic glasses.
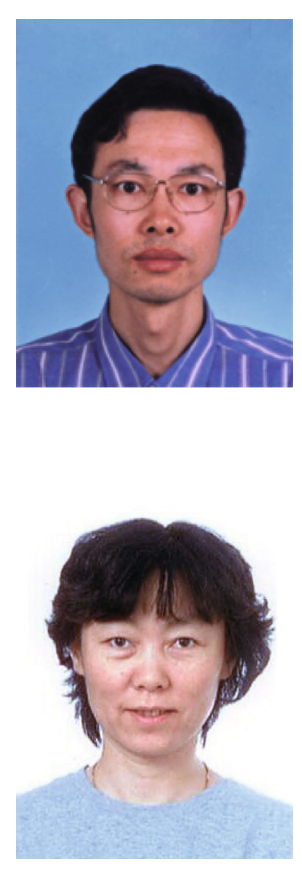

Wei-Hua Wang is a professor of the Institute of Physics, Chinese Academy of Sciences at Beijing, China. He earned his PhD degree in condensed matter physics at Chinese Academy of Sciences in 1993, and was Humboldt fellow during 1995-1997. His research focuses on the formation, structure, physical properties and glass transition in metallic glasses.

Hai-Yang Bai is a professor of the Institute of Physics, Chinese Academy of Sciences at Beijing, China. She received a $\mathrm{PhD}$ degree in physics from Institute of Physics in 1991, and was Humboldt fellow during 1994-1997. Her scientific interests are focused on the thermodynamics, structure, and mechanical properties in metallic glasses.

中文摘要 形成非晶的过冷液体在接近玻璃转变温度的动力学响应的多样性和非均匀性可以遗传到非晶玻璃态中, 表现为结构非均匀 性. 这种结构非均匀性可以通过其应力弛豫的不同来表征. 在此实验结果基础上, 本文提出了一个可以容易地通过应力弛豫测量的参 数 $n$, 以此参数来表征不同非晶合金的动力学和结构非均匀性的差异, 参数 $n$ 还能反映非晶体系的流变单元的密度和分布, 因此可以用 $n$ 来对不同脆度的非晶玻璃进行分类. 研究还发现该参数 $n$ 还和过冷液体动力学脆性参数 $m$ 以及非晶泊松比有关, 可能成为表征金属玻璃 的动力学和结构非均匀、结构和性能关系, 非晶本质的重要参数. 